\title{
From Data to Models and Back
}

\author{
Mike Dreves \\ mdreves@google.com \\ Google Inc. \\ Neoklis Polyzotis \\ npolyzotis@google.com \\ Google Inc.
}

\author{
Gene Huang \\ jinhuang@google.com \\ Google Inc. \\ Evan Rosen \\ embr@google.com \\ Google Inc.
}

\author{
Zhuo Peng \\ zhuo@google.com \\ Google Inc.
}

\author{
Paul Suganthan G. C. \\ pachristopher@google.com \\ Google Inc.
}

\begin{abstract}
Production ML is more than writing the code for the trainer. It requires processes and tooling that enable a larger team to share, track, analyze, and monitor not only on the code for ML but also on the artifacts (Datasets, Models, ...) that are manipulated and generated in these production ML pipelines.

In this paper we describe the tools we developed at Google for the analysis and validation of two of the most important types of artifacts: Datasets and Models. These tools are currently deployed in production at Google and other large organizations. Our approach is heavily inspired by well-known principles of data-management systems. Ultimately, we want to enable users to trust their data and models, and understand how data properties affect the quality of the generated ML models.
\end{abstract}

\section{ACM Reference Format:}

Mike Dreves, Gene Huang, Zhuo Peng, Neoklis Polyzotis, Evan Rosen, and Paul Suganthan G. C.. 2020. From Data to Models and Back. In International Workshop on Data Management for End-to-End Machine Learning (DEEM'20), fune 14, 2020, Portland, OR, USA. ACM, New York, NY, USA, 4 pages. https://doi.org/10.1145/3399579.3399868

\section{INTRODUCTION}

ML has succeeded in tackling a wide range of challenging problems in practice, from medical applications (e.g., the detection of retinopathy [2]), to self-driving cars, or agriculture [3], to name a few cases. Moreover, there is a fast pace of innovation in the scientific field of ML.

When one begins to use ML, they naturally think about writing the code that can train a model based on input data. This is an important step of using ML, but this code is only a small piece of what it takes to run ML reliably. In a production setting, the user of ML has to worry about: whether the input data has errors; how to trigger a retraining of the model when new data arrives; whether the new version of a model is good enough to replace the model currently used by the downstream stack; whether the serving data (used for prediction requests to the model) is sufficiently different such that retraining is required; and many more issues.

Permission to make digital or hard copies of part or all of this work for personal or classroom use is granted without fee provided that copies are not made or distributed for profit or commercial advantage and that copies bear this notice and the full citation on the first page. Copyrights for third-party components of this work must be honored.

For all other uses, contact the owner/author(s).

DEEM'20, fune 14, 2020, Portland, OR, USA

(C) 2020 Copyright held by the owner/author(s)

ACM ISBN 978-1-4503-8023-2/20/06.

https://doi.org/10.1145/3399579.3399868
At Google, we refer to this set of concerns as "ML engineering" in order to separate them from "ML coding", which refers to the relatively smaller task of writing the trainer. In using this term we draw an analogy to the distinction between software engineering and coding. Usually, coding refers to a one-off, monolithic piece of code that is not meant for sharing, whereas software engineering establishes processes and practices around versioning, testing, and so on, to enable a larger team to work on a shared code base that solves a bigger problem. The same applies to ML engineering: we need processes and tooling around versioning, testing, monitoring, and so on, that apply not only to code but also to ML artifacts such as datasets and models.

In this paper, we describe the tooling and processes we developed for the analysis and validation of datasets and models. These are two of the most important artifacts in production ML pipelines, with clear connections to the overall effectiveness of ML. We adopt a data-oriented approach to the management of these artifacts. This is obvious for dataset artifacts. For model artifacts, we note that the quality of a model is inherently tied to the data used for training, evaluation, and serving. Moreover, with the advent and maturation of Auto-ML solutions which can optimize the parameters of the model's architecture, the input data becomes the next obvious knob that can be tweaked to improve model quality. Thus, an approach based on data-management principles can be usefully extended to models.

Our work is in the context of TensorFlow Extended (TFX), a production-ML platform that we developed for Google's product teams and the world. The design and functionality of our tools is heavily influenced by our experience with ML in Google's production setting, but we believe that the basic principles and lessons learned apply to other contexts, and are of interest to both researchers and practitioners.

\section{DATA ANALYSIS AND VALIDATION}

ML exemplifies the "garbage-in, garbage-out" principle: if the input data has errors then it is unlikely that we will get a model of high quality. Hence, much like we need unit tests to check that a piece of code meets some expected behaviors, we need the equivalent of "data tests" to ensure that the data has some expected properties that make it likely we can train good models. For this purpose, we developed TensorFlow Data Validation (TFDV) [4], a scalable data analysis and validation system for ML. We cover the salient features of TFDV in what follows, and defer the technical details to the corresponding paper [8].

In order to codify the expected data characteristics, TFDV generalizes the traditional notion of a schema from database systems. The 
schema follows a logical data model where each training or serving example is a collection of features, with each feature having several constraints attached to it. The constraints associated with each feature cover some basic properties (e.g., type, domain, valency) but also constraints that are relevant to ML.

TFDV checks the properties of the data as specified via the schema. Specifically, given a batch of data, TFDV computes a predefined set of data statistics which is validated against the schema. Any deviation within a batch from the schema is considered an anomaly. TFDV supports two types of validation: (1) validating a single batch of data relative to the schema, and (2) validating two batches of data relative to one another (e.g., are there any significant changes between training and serving data, or between successive batches of the training data?).

TFDV can also identify mismatches between the expected data and the assumptions made in the training/serving code. This helps answer the question: are there any assumptions in the training/serving code that are not reflected in the data? (E.g. are we taking the logarithm of a feature that turns out to be a negative number or a string?). We base our approach on fuzz testing, using the schema to guide the generation of synthetic inputs. Specifically, we generate synthetic training examples that adhere to the schema constraints. The generated data is then used to drive a few iterations of the training code. The goal is to trigger any hidden assumptions in the code that are inconsistent with the schema constraints.

Our system is deployed in production as an integral part of TFX [7], an end-to-end ML platform, and is used by hundreds of product teams at Google to monitor and validate trillions of training and serving examples per day, amounting to several petabytes of data per day. We recently open sourced TFDV and the system has received significant attention from the open-source community as well: $2.6 \mathrm{M}$ downloads since the first release in October 2018, with documented usage in other large organizations [6]. Moreover, TFDV has influenced the development of other open-source data validation systems such as Apache Spark Data Validation [1].

Few recent works ([14], [11]) have considered the importance of data validation for ML applications. For example, Amazon (Schelter et. al. [14]) proposed a system for automating data quality verification task that provides a declarative API to specify common quality constraints and custom validation code. While Amazon's system allows users to express arbitrary constraints, we opted to have a restrictive schema definition language that captures the data constraints for most of our users in order to focus on reliable high precision alerts. Further our work emphasizes on the co-evolution of the schema with data and the model with the user in the loop. As far as we can tell, TFDV is the first open-source data analysis and validation system for ML.

\section{MODEL ANALYSIS AND VALIDATION}

After training a model based on (validated) input data, the next step in a production pipeline is analyzing the trained model and deciding whether it can be pushed to the inference/serving stack. This step makes the model available to other applications. However, pushing a model that returns sub-optimal or even erroneous predictions can lead to undesired downstream effects. As an example, if a bad model generates uninteresting or irrelevant recommendations in a movie-streaming service the user experience is likely to suffer. What is needed is the equivalent of an integration test, where we can evaluate the model's accuracy on unseen data. For this purpose, we have developed TensorFlow Model Analysis (TFMA) [5].

While data validation prevents errors through inappropriate input data, it is still possible to introduce errors into the pipeline as a result of improper training. Most ML frameworks provide tools for evaluating metrics of interest (e.g., loss or AUC) during training. TFMA takes this a step further by allowing users to re-evaluate their models post-training over large amounts of data in a distributed manner. This evaluation can be done using the same metrics that were defined during training or with additional metrics added after the model has been generated. While TFMA performs full passes over generally much larger data than is seen at training time, the datasets are still just samples drawn from a larger population. To help gauge the reliability of these computations, TFMA provides confidence intervals for the metrics it computes. For increased reliability and safety, TFMA provides a means to set expectations on model performance metrics using either absolute thresholds or thresholds relative to a baseline. Practitioners can then gate pushing their models to production based on passing the said validation thresholds. In a sense, this model-based validation complements the data-based validation implemented by TFDV.

TFMA also supports computing and validating model metrics on data slices. Model metrics computed on the whole evaluation dataset can mask interesting or significant deviations of the same metrics computed on data slices that correspond to meaningful sub-populations. For instance, a machine-translation model may perform adequately well on average but significantly worse on a specific language. TFMA allows users to declare slices of interest and then to get a more detailed view of model metrics computed on the corresponding subsets of the evaluation data. Model validation can subsequently verify these metrics in order to guard against model regressions which only affect a small but important slice of the evaluation data.

In many cases, users do not know a priori which slices are important. Alternatively, a user might miss important sub-slices within the manually defined slices (e.g., the machine-translation model may under-perform for a specific language only when the translation request comes from a specific class of devices). To aid with these cases we investigated techniques to automatically identify slices of interest [10]. We view this automatic slicing as a complement to the manual slicing already supported by TFMA. For instance, it is possible to start with manually defined slices and then automatically discover subslices of interest. Our inspiration in this domain has been previous works in discovering interesting roll-ups/drill-downs in data cubes $[12,13,15]$. Moreover, recent work [9] in the ML community has shown how knowledge about under-performing slices can be leveraged in order to improve overall model quality.

\section{CONNECTING STRUCTURED DATA TO ML}

A common assumption in ML frameworks is that the data needs to be translated to some intermediate "container" format before it can be used for training or model evaluation. For instance, TensorFlow supports the $t f$. Example $e^{1}$ format which represents each data

\footnotetext{
${ }^{1}$ https://www.tensorflow.org/api_docs/python/tf/train/Example
} 
model.py (in training)

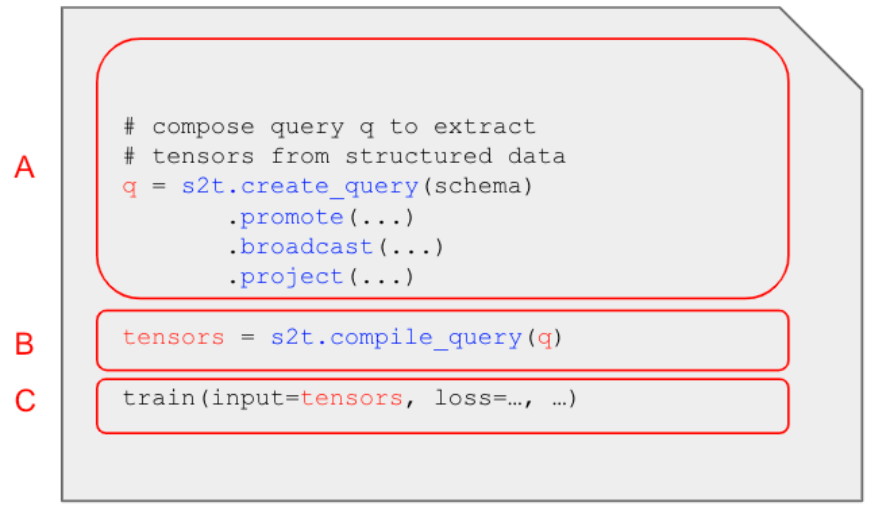

Trained model

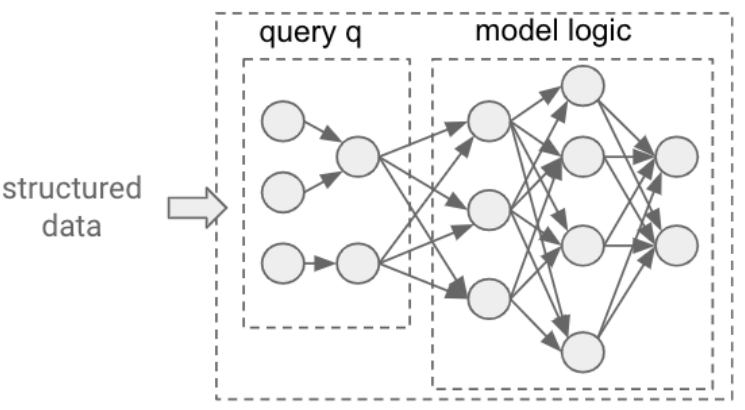

Figure 1: Using struct2tensor to provide structured data as input to a model. (A) the user writes a declarative query that translates the structured data to Tensors; (B) the query is compiled and optimized into a graph of TensorFlow ops; (C) the graph is used as the first (input) layer for the model. The resulting model topology is shown on the right, showing the query mini-graph at the beginning of the model.

record as a collection of key-value pairs corresponding to features and their values.

These intermediate formats are convenient in that they define a "data" API that decouples the ML framework from the datagenerating pipelines. However, most teams start with data in different, application-specific formats, which means that a data translation step is necessary before it is possible to train on the data, or use it to evaluate a model or obtain predictions from a model. This creates several issues.

First, this data conversion requires extra code and effort and thus raises the bar for using ML. Moreover, any experimentation with additional features from the raw data requires writing more code and re-running the conversion pipeline, which limits velocity for experiments. This data translation also needs to happen consistently when training a model and also when feeding data for predictions. In many cases training and serving follow different code paths, and any inconsistencies in the translation lead to training/serving skew that can impact model quality. Another issue is that our tooling (TFDV and TFMA) can only analyze the converted data, which means that the user is left with the task to map the analysis results back to the original data. For instance, if TFDV discovers errors on some feature $\mathrm{X}$ in the input tf.Examples, then the user has to map this error back to the fields of the raw data in order to fix it. Depending on the complexity of the data-conversion pipeline, this can be a difficult task. Finally, all of these issues are exacerbated as the complexity of these container formats increases. For instance, there are ML libraries that can accept data formatted as tf.SequenceExample or tf.ExampleListWithContext, two container formats that add structure on top of the flat data of tf.Example. This increase in complexity, driven by more powerful ML algorithms that can leverage structure in the training data in order to generate more effective models, is likely to continue.
To address these issues we developed a solution, named struct2tensor, that allows TensorFlow and our tooling to directly manipulate structured data. The idea behind struct2tensor comes from the concept of logical views. Specifically, we allow ML users to write declarative queries over their structured data that output a Tensor-based view suitable for processing through TensorFlow training and serving. In turn, the view becomes the very first stage of the model, which means that the model now expects structured data as input (with the translation to Tensors happening inside the model).

Figure 1 shows a schematic of the end-to-end flow: the user writes the declarative query as part of their Python code for the trainer, the query is compiled into a graph of TF.Ops, and the graph is inlined with the model. This approach brings numerous advantages: users do not need to convert their data and can immediately start experimenting with ML; extracting new features means simply changing the query, so new experiments become easier; data translation occurs hermetically inside the model, and so can happen consistently for training and serving (i.e., no skew); since the user accepts structured data as input, data and model analysis can occur directly on the data that the user is most familiar with, without the burden of translating the analysis between formats; and, by making models accept structured data directly as input, we unlock the potential of powerful ML algorithms that can leverage this structure.

Since struct2tensor operates on the inference path (to translate a structured data record into tensors in order to obtain a prediction) it is imperative that it works with low latency. For this purpose, we incorporated several optimization strategies in the translation of the declarative query to a graph of TensorFlow Ops (step B). Some of these optimizations are specific to TensorFlow, but others resemble common tricks from relational query optimizers, e.g., predicate reordering, projection push-down, and selective parsing. 
As mentioned earlier, there are several similarities between struct2tensor and relational views. There are also important differences: the input and output data models are different (structured data vs tensors); the query language is specific to the type of data transformations that occur in feature extraction for ML; and, the execution engine for view evaluation is TensorFlow, which affects the cost factors (and hence the optimizations that we can apply) of the physical execution plans.

We have deployed struct2tensor at Google in large production models that receive millions of QPS. These successful deployments have helped us validate the stated benefits of our view-based approach, and they also verified the effectiveness of our optimizations in making struct2tensor efficient for the serving path. We also observed a side-benefit which we did not anticipate but in hindsight became obvious: by obviating the need to translate data to these intermediate formats (specifically, tf.Example in our case), we allowed product teams to save on storage space, which in turn allowed them to gather more training data and use it to improve the quality of their models! In fact, the savings in storage space were more than $2 x$, since these container formats are not optimized for storage efficiency.

\section{REFERENCES}

[1] [n.d.]. Apache Spark Data Validation. https://databricks.com/session/apachespark-data-validation

[2] [n.d.]. Diagnosing Diabetic Retinopathy with Machine Learning. https://about. google/intl/en_us/stories/seeingpotential
[3] [n.d.]. How a Japanese cucumber farmer is using deep learning and TensorFlow. https://cloud.google.com/blog/products/gcp/how-a-japanese-cucumberfarmer-is-using-deep-learning-and-tensorflow

[4] [n.d.]. TensorFlow Data Validation. https://github.com/tensorflow/datavalidation

[5] [n.d.]. TensorFlow Model Analysis. https://www.tensorflow.org/tfx/model_ analysis/get started

[6] [n.d.]. The Winding Road to Better Machine Learning Infrastructure Through Tensorflow Extended and Kubeflow. https://labs.spotify.com/2019/12/13/thewinding-road-to-better-machine-learning-infrastructure-through-tensorflowextended-and-kubeflow/

[7] Denis Baylor, Eric Breck, Heng-Tze Cheng, et al. 2017. TFX: A TensorFlow-Based Production-Scale Machine Learning Platform. In ACM SIGKDD.

[8] Eric Breck, Marty Zinkevich, Neoklis Polyzotis, Steven Whang, and Sudip Roy. 2019. Data Validation for Machine Learning. In SysML.

[9] Vincent Chen, Sen Wu, Alexander J Ratner, Jen Weng, and Christopher Ré. 2019. Slice-based Learning: A Programming Model for Residual Learning in Critical Data Slices. In Advances in Neural Information Processing Systems 32, H. Wallach, H. Larochelle, A. Beygelzimer, F. d'Alché-Buc, E. Fox, and R. Garnett (Eds.). Curran Associates, Inc., 93979407. http://papers.nips.cc/paper/9137-slice-based-learning-a-programmingmodel-for-residual-learning-in-critical-data-slices.pdf

[10] Y. Chung, T. Kraska, N. Polyzotis, K. H. Tae, and S. E. Whang. 2019. Slice Finder: Automated Data Slicing for Model Validation. In 2019 IEEE 35th International Conference on Data Engineering (ICDE). 1550-1553.

[11] Stephan Rabanser, Stephan Günnemann, and Zachary Lipton. 2019. Failing Loudly: An Empirical Study of Methods for Detecting Dataset Shift. In Advances in Neural Information Processing Systems 32.

[12] Sunita Sarawagi. 2000. User-adaptive exploration of multidimensional data. In VLDB, Vol. 2000. 307-316.

[13] Gayatri Sathe and Sunita Sarawagi. 2001. Intelligent rollups in multidimensional OLAP data. In $V L D B$, Vol. 1. 531-540.

[14] Sebastian Schelter, Dustin Lange, Philipp Schmidt, et al. 2018. Automating LargeScale Data Quality Verification. VLDB 11, 12 (2018), 1781-1794.

[15] Manasi Vartak, Samuel Madden, Aditya Parameswaran, and Neoklis Polyzotis. 2014. SeeDB: Automatically Generating Query Visualizations. Proc. VLDB Endow. 7, 13 (Aug. 2014), 1581-1584. https://doi.org/10.14778/2733004.2733035 